\title{
A 3-V CMOS OPTICAL PREAMPLIFIER WITH DC PHOTOCURRENT REJECTION
}

\author{
K. Phang and D. A. Johns \\ Dept. of Electrical and Computer Engineering \\ University of Toronto \\ Toronto, Ontario, M5S 3G4, CANADA
}

\begin{abstract}
This paper describes a CMOS optical preamplifier suitable for free-space, infrared wireless communications. The design is differential for improved power supply noise rejection, and an active cancellation scheme is used for eliminating dc photocurrents that are generated by ambient light. Making use of a $0.35-\mu \mathrm{m}$ CMOS process, the preamplifier provides a transimpedance gain of $20 \mathrm{k} \Omega$ over a bandwidth from $1 \mathrm{MHz}$ to $100 \mathrm{MHz}$ and achieves $60 \mathrm{~dB}$ attenuation at dc.
\end{abstract}

\section{INTRODUCTION}

The proliferation of infrared (IR) wireless technology in laptops, computer peripherals, and digital cameras has helped drive new developments in the area of optical receiver design. Traditional applications such as fiber-optic networks have emphasized speed, and consequently have used high-speed processes such as GaAs. IR wireless, on the other hand, emphasizes system integration and cost. Consequently, bulk CMOS is the preferred technology. Recent papers on CMOS optical preamplifiers[1-3] have highlighted some of the design challenges of single-chip receivers, specifically that of obtaining sufficient speed and sensitivity given low supply voltages, small transconductances, and noisy power supplies.

Because of its wide bandwidth and low noise, the transimpedance amplifier is commonly used in optical receivers[4]. Although inverter-based transimpedance amplifiers have shown good speed and sensitivity[2], they generally have poor power supply rejection because of the capacitive coupling of the supplies to the signal path[5]. A more robust approach is to use a differential structure with shunt feedback[3]. However, this approach has only been realized using bipolar and NMOS devices. This paper describes how the structure can be adapted and optimized for a true CMOS implementation.

Apart from the preamplifier, IR wireless communications itself poses numerous challenges. One major concern is the presence of dc photocurrents generated when a photodiode is exposed to ambient indoor light. Often the dc photocurrent is much larger than the signal current, resulting in reduced signal swing or even saturation. One solution is to ac couple the photodiode to the preamplifier input[6]. However, depending on the desired high-pass frequency, large capacitors and resistors may be required. For monolithic implementations, this implies increased chip area and sensitivity to parasitic-coupled noise. Consequently, we propose instead to use an active cancellation scheme for dealing with dc photocurrents.

\section{BASIC ARCHITECTURE}

The concept of active dc photocurrent rejection is illustrated in Fig. 1. It involves adding a variable current source represented here by the transistor, Mctl, to draw the dc current, Idc, from the photodiode away from the preamplifier input. A feedback loop which includes an error amplifier is used to determine the optimum current level. Similar structures using peak-level detection have been reported [7-8]. However, by modifying the feedback loop to use simple low pass filtering instead of peak detection, the reset and control circuitry -- possible sources of digital noise -- are eliminated. The resulting circuit requires little area and allows for the tuning of the low frequency cut-off point.

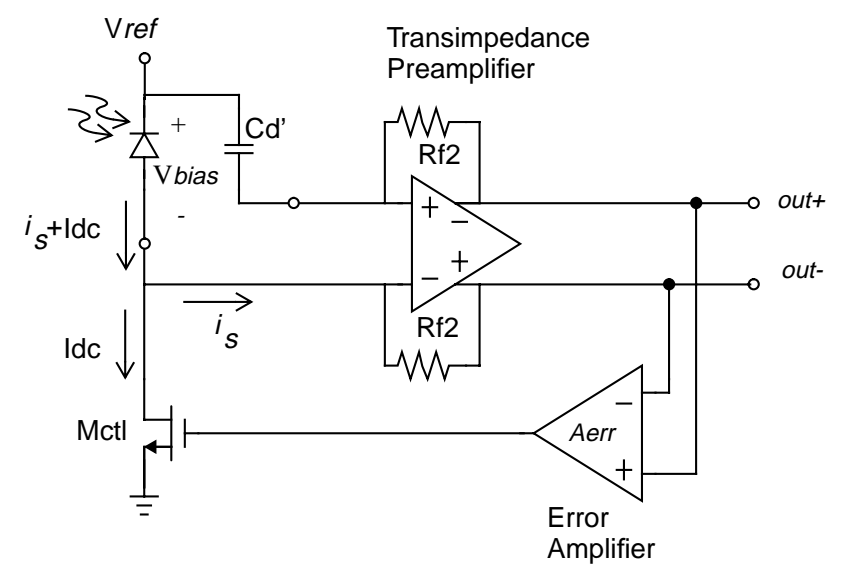

Fig. 1 Optical preamplifier with dc photocurrent cancellation.

The signal path is made differential in order to maximize the preamplifier's immunity to noise from the power supply. Noise from the bias voltage, Vref, is also a concern as it is coupled to the preamplifier input through the depletion capacitance of the photodiode. In order to help cancel this noise, we propose adding a matching capacitance, $C d$ ', from Vref to the opposite input terminal; given perfect matching, the noise should appear only as a common-mode signal that is effectively suppressed by the balanced structure.

The next section discusses the design of the transimpedance amplifier, followed by an analysis of the feedback loop used for dc photocurrent rejection. 


\section{BALANCED TRANSIMPEDANCE AMPLIFIER}

The transimpedance amplifier, along with a model of the photodiode, is shown in Fig. 2. The circuit has a balanced structure consisting of an input differential pair followed by a pair of common-source second stages that utilize shunt feedback. Although, the basic structure follows the design reported by Coppoolse et al.[3], significant changes were made to adapt their original $5-\mathrm{V}$ design for $3-\mathrm{V}$ operation. Specifically, we reduced the number of stages from three to two, replaced all biasing resistors with active current sources, and introduced a p-type input stage to create a complementary structure.

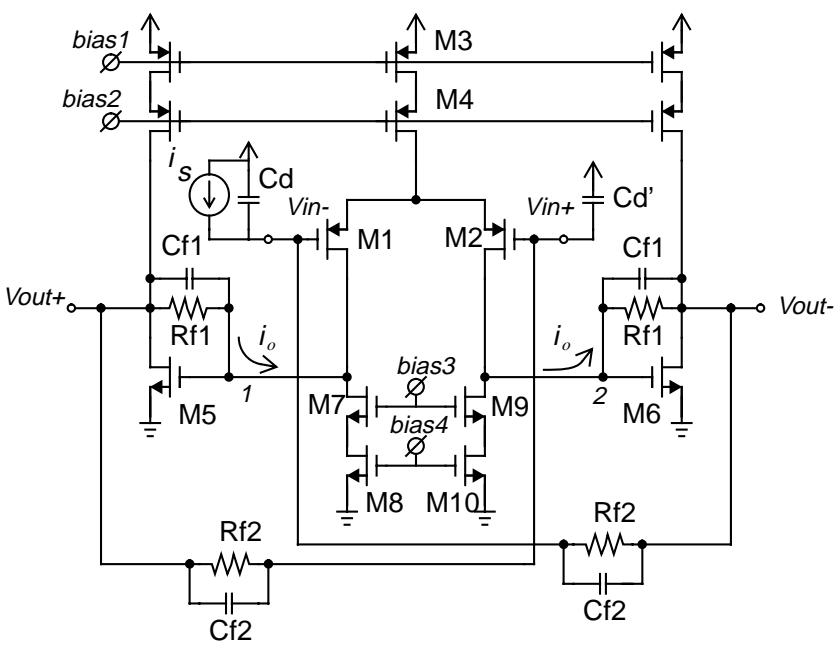

Fig. 2 Balanced transimpedance amplifier with shunt feedback.

The transimpedance amplifier uses a p-type input stage for a number of reasons. Because the depletion capacitance of a photodiode is inversely proportional to its reverse bias voltage, Vbias, high speed is achieved by maximizing Vbias. For example, increasing Vbias from $1-\mathrm{V}$ to $2-\mathrm{V}$ on the Temic BPV-10NF PIN photodiode changes the depletion capacitance from $6.6 \mathrm{pF}$ to $5.2 \mathrm{pF}$-- a significant $20 \%$ reduction. With a p-type differential pair, the inputs can be set near ground to maximize Vbias without fear of saturation. In addition, setting the input levels low ensures a large voltage drop across the tail current source, allowing the use of cascode devices to improve current matching between the tail current and the active loads. Good matching is important in this design because the lack of common-mode feedback implies any mismatch in the currents will be fed into the second stage, producing an offset at the output. Finally, p-type inputs necessitate a n-type second stage which is beneficial since the higher mobility of n-type devices help to place the non-dominant poles of the circuit higher in frequency, allowing faster operation and improved stability.

Two interesting properties resulting from the local feedback are the circuit's inherent low output impedance and the natural biasing of the output common-mode level. The low output impedance can be deduced by realizing that devices M5 and M6 are now diode-connected through their respective $R_{f 1}$ resistors. Assuming that $R_{f 1}$ is not extremely large, the output impedance is equal to the inverse of the transconductances of M5 and M6 which is on the order of a few hundred ohms. A low output impedance allows us to eliminate the output source follower stage used in [3] to achieve an improved phase response. The output common-mode voltage is naturally set to $V_{\mathrm{gs} 5,6} \approx 0.9 \mathrm{~V}$.

Having explained the basic structure of the transimpedance amplifier, we now present the main design equations. Although the photocurrent signal is single-ended, the final output is differential; therefore, the circuit's differential behavior is considered when characterizing the signal path. The transimpedance gain of the circuit is given by

$$
\begin{aligned}
\frac{v_{\text {out }+}-v_{\text {out- }}}{i_{s}} & =\frac{A v d}{1+A v d} R_{f 2} \\
& \approx R_{f 2} \quad \text { for } A v d » 1
\end{aligned}
$$

where $A v d$ is the open-loop voltage gain of the amplifier. By symmetry, Avd can be determined by analyzing the left half-circuit of Fig. 2. Because the second stage has a low input impedance roughly equal to the inverse of the transconductance, $g_{m 5}$, the intermediate current from the differential pair, $i_{o}$, is effectively steered into resistor $R_{f 1}$. Small-signal analysis reveals that the second stage has a transimpedance of

$$
\frac{v_{\text {out }+}}{i_{o}}=-\frac{\left(1-g_{m 5} R_{f 1}\right) r d s_{5}}{1+g_{m 5} r d s_{5}} \approx R_{f 1} .
$$

The overall gain is the product of the two gain stages:

$$
\begin{aligned}
A v d & =\frac{v_{\text {out }+}}{v_{i n-}}=\frac{v_{\text {out }+}}{i_{o}} \times \frac{i_{o}}{v_{i n-}} \\
& \approx g_{m 1} R_{f 1}
\end{aligned}
$$

which is typically in the range of 10 to a few hundred.

The procedure for optimizing the circuit for a given bandwidth involves analyzing the loop gain. Because the feedback network consists of $R_{f 2}$ in series with photodiode depletion capacitance, $C_{d}$, the dc loop gain is equal to the differential voltage gain given in equation (3). Because $C_{d}$ is typically quite large (e.g. $\sim 5 \mathrm{pF}$ ), the dominant pole is located at the input and is given by

$$
w_{p 1}=\frac{-1}{R_{f 2} C_{d}} .
$$

From Equations (3) and (4), it is clear that the dominant pole is independent of the dc loop gain. As a result, the bandwidth of the circuit is proportional to $A v d$, and in order to increase the bandwidth, both $g_{m 1}$ and $R_{f 1}$ should be maximized. While transconductance $g_{m 1}$ is ultimately limited by power constraints, resistance $R_{f 1}$ is limited by the resistor's effect on the non-dominant poles in the second stage. Small-signal analysis reveals that a pair of complex poles are introduced by the second stage. In practice, the feedback capacitor, $C_{f 1}$, is relatively small compared to both the total capacitance on node- $1, C_{1}$, and the total capacitance at the output node, $C_{\text {out+ }}$. The resulting pole 
locations are given by

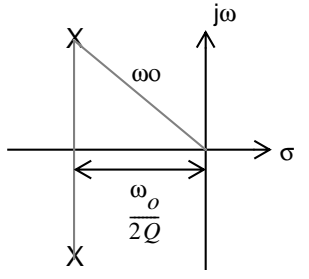

$$
\begin{aligned}
& \omega_{o} \approx \sqrt{\frac{g_{m 5}}{C_{\mathrm{out}+} C_{1} R_{f 1}}} \\
& Q \approx \frac{1}{C_{f 1}} \sqrt{\frac{C_{\mathrm{out}+} C_{1}}{g_{m 5} R_{f 1}}} .
\end{aligned}
$$

From Equation (5), we see that the upper limit of $R_{f 1}$ is set by the desired bandwidth of the circuit: to ensure stability, the unity loop gain frequency should not exceed $\omega_{o}$. It is useful to note that while $\omega_{o}$ is inversely proportional to the square root of $R_{f 1}$, the dominant pole, $\omega_{p 1}$, is inversely proportional to $R_{f 2}$. Thus if during implementation, $R_{f 2}$ could be made to track $R_{f 1}$, stability is insured because the dominant pole would drop faster than the non-dominant pole if ever $R_{f .1}$ increased past its nominal value. We also see from equation (6) that capacitor $C_{f 1}$ plays a dominant role in determining the Q-factor of the poles. Thus, $C_{f 1}$ can be used to tailor the response of the second stage to have a maximally-flat frequency response.

The other feedback capacitor, $C_{f 2}$, also serves to tailor the overall frequency response by introducing a zero in the left-half plane at

$$
\omega_{z}=\frac{-1}{R_{f 2} C_{f 2}} .
$$

This zero compensates for the $90^{\circ}$ phase lag that is generated by the series $R_{f 2}-C_{d}$ feedback network.

To illustrate the design process using a $0.35-\mu \mathrm{m}$ CMOS technology, we optimized the transimpedance amplifier for a bandwidth of $100 \mathrm{MHz}$ while limiting the power dissipation to $10 \mathrm{~mW}$. For the given power, we achieve a transconductance of $4.4-\mathrm{mA} / \mathrm{V}$ for the first stage, and 3.6-mA/V for the second stage. From equation (5), we find the largest $R_{f 1}$ value to be $20 \mathrm{k} \Omega$ which places the complex set of poles at around $136 \mathrm{MHz}$, sufficiently beyond the $100-\mathrm{MHz}$ bandwidth. By setting $C_{f 1}=60 \mathrm{fF}$, we achieve a Q-factor of 0.8 which produces a nearly maximally-flat response for the second stage. To determine the optimum value of $R_{f 2}$ for a $100 \mathrm{MHz}$ bandwidth, we first calculate the dc loop gain which turns out to be 72 . If we set the gain-bandwidth to $100 \mathrm{MHz}$, the dominant pole, $\omega_{p 1}$, should be located at $100 / 72 \mathrm{MHz} \approx 1.3 \mathrm{MHz}$. Substituting a photodiode capacitance of $5 \mathrm{pF}$ into Equation (4), $R_{f_{2}}$ is determined to be $23 \mathrm{k} \Omega$ and so we round it down to $20 \mathrm{k} \Omega$ in order to equal $R_{f 1}$. Finally, fine tuning the zero location for lead compensation, we set $C_{f 1}$ to $60 \mathrm{fF}$. Simulation waveforms of the transimpedance amplifier are shown in Fig. 3; the preamplifier has a bandwidth of $120 \mathrm{MHz}$ and an input-referred noise current of $1.3 \mathrm{pA} / \sqrt{\mathrm{Hz}}$. These results are favorable compared with those presented in [3]: with a technology about four times faster, we achieve over twice the gain and our bandwidth is only about $40 \%$ lower even though the photodiode capacitance is over ten times larger. Having completed the design of the transimpedance amplifier, we are ready to incorporate it into the dc photocurrent rejection circuit.
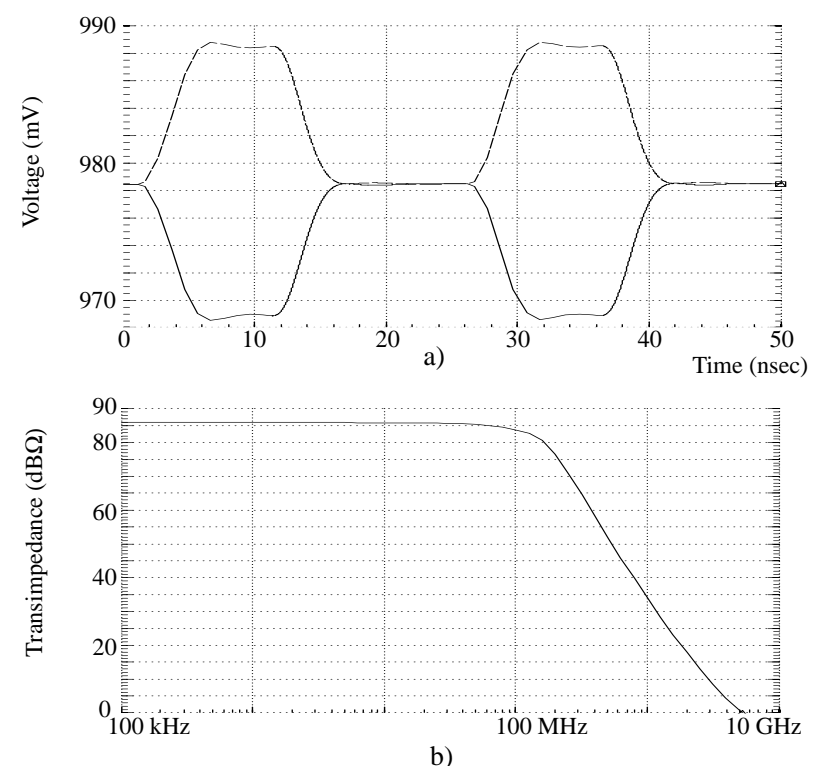

Fig. 3 Simulation results of the transimpedance amplifier:

a) Differential outputs for a $1 \mu \mathrm{A}$ input pulse

current, b) Transimpedance frequency response.

\section{DC PHOTOCURRENT REJECTION}

As described earlier, our dc photocurrent rejection circuit uses negative feedback to minimize the average differential output voltage. Fig. 4a) shows representative differential outputs from the preamplifier when no dc photocurrent is present and the active cancellation is off. When a dc component is added, the outputs shift away from each other as in Fig. 4b), resulting in an positive, common-mode offset. When the active cancellation is enabled, the positive offset causes the error amplifier to raise the gate voltage of transistor, Mctl, causing the device to begin drawing the $\mathrm{dc}$ component away from the preamplifier input. When the outputs have settled, both the offset due to the dc photocurrent as well as the average signal level have been eliminated as in Fig. 4c).

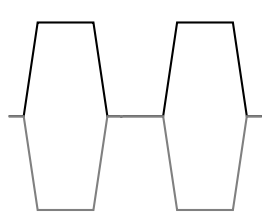

a)
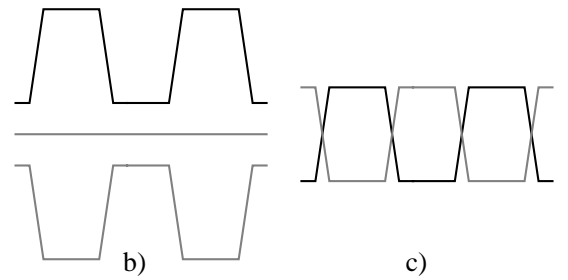

c)
Fig. 4 Differential output waveforms: a) no dc photocurrent, b) dc photocurrent added, c) steady-state.

The elimination of the average signal current is not a concern so long as the low-frequency cut-off point is sufficiently below the signal spectrum. For our system we have specified a cut-off frequency of $1 \mathrm{MHz}$ which is sufficiently low for a $100 \mathrm{MHz}$ bandwidth, and adequately high to attenuate the fluctuating light levels produced by artificial light sources, and in particular, fluorescent lamps 


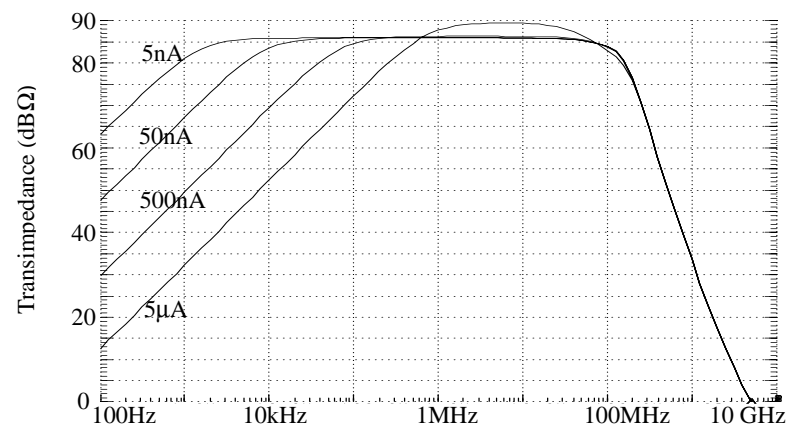

Fig. 5 Frequency response of preamplifier with dc photocurrent rejection for different current levels.

with electronic ballasts[9].

The characteristics of the dc photocurrent rejection circuit can be derived by studying the loop gain of the outer feedback loop. The residual dc photocurrent entering the preamplifier is inversely proportional to the open-loop gain at dc. As well, the low frequency cut-off point, $\omega_{\text {cut_off }}$, is given by the unity loop-gain frequency. The open-loop gain, $L(s)$, is given by

$$
\begin{aligned}
L(s) & =g_{m c t l} R_{f 2} A_{e r r}(s) \\
& =K_{c t l} \sqrt{I d c} R_{f 2} A_{e r r}(s)
\end{aligned}
$$

where $A_{\text {err }}(s)$ is the frequency response of the error amplifier, and constant, $K_{c t l}$, is determined by the geometry of Mctl and the CMOS process parameters. Because the bandwidth of this feedback loop is low, the frequency response of the error amplifier can be modeled using a single-pole response of

$$
A_{\text {err }}(s)=\frac{A o}{1+s / \omega_{\text {err }}}
$$

where $\omega_{\text {err }}$ is the dominant-pole frequency and Ao is the $\mathrm{dc}$ gain. The gain-bandwidth product of this circuit is

$$
\left|L\left(j \omega_{\text {err }}\right)\right| \times \omega_{\text {err }}=1 \times \omega_{\text {cut_off }}
$$

which leads to the following relationship between the low frequency cut-off point and the dc photocurrent:

$$
\omega_{\text {cut_off }}=\omega_{\text {err }} K_{c t l} R_{f 2} A o \sqrt{I d c}
$$

The main observation from Equations (8) and (11) is that both the open-loop gain and cut-off frequency are affected by the dc photocurrent level. Fig. 5 shows the simulated frequency response of the circuit incorporating our optimized transimpedance amplifier. From $5 \mu \mathrm{A}$ down to $5 \mathrm{nA}$, the curves show how decreasing the dc photocurrent degrades the regulation while shifting down the cut-off frequency. Yet this situation is not as onerous as it would first appear once we look at the possible situations. True, the dc regulation is poor when there is little photocurrent, but when there is little photocurrent, dc regulation is not required; when the dc photocurrent level is high, so is the effective regulation. Thus, the feedback loop performs well in situations where it is truly needed.
The dependency of the cut-off frequency on the dc photocurrent does pose a concern in the case of large photocurrents. For instance, from Fig. 5 we see that beyond 500-nA, the cut-off frequency exceeds our $1 \mathrm{MHz}$ specification, and at $5 \mu \mathrm{A}$, the loop gain has increased to the point that it begins affecting the passband response of the transimpedance amplifier. Ideally, we would like some method of limiting the photocurrent which passes through transistor Mctl. A potential solution would be to redirect the excess photocurrent into another current source that is static, but which can be switched on when needed. In this way the new current source does not change the loop dynamics, but simply off-loads some of the current transistor Mctl would otherwise need to draw.

\section{SUMMARY}

This paper proposes an optical receiver structure for use in infrared wireless communications. The structure actively rejects dc photocurrents using negative feedback around the core transimpedance amplifier. The rejection performance was shown to improve with increasing photocurrent levels.

The differential, shunt-feedback transimpedance amplifier exhibits good performance, and we have shown how it can be adapted for low-voltage CMOS technology. Simulation results show the preamplifier provides a gain of $20 \mathrm{k} \Omega$ over a bandwidth from $1 \mathrm{MHz}$ to $100 \mathrm{MHz}$ with an input noise current density of $1.3 \mathrm{pA} / \sqrt{\mathrm{Hz}}$. The optical preamplifier is currently being fabricated using a $0.35 \mu \mathrm{m}$ CMOS process.

\section{REFERENCES}

[1] M. Nakamura et al., "An Instantaneous Response CMOS Optical Receiver IC with Wide Dynamic Range and Extremely High Sensitivity Using Feed-Forward Auto-Bias Adjustment," IEEE J. Solid-State Cir., Vol. 30, No. 9, pp. 991-997, Sept. 1995.

[2] M. Ingels, G. Van der Plas, J. Crols, and M. Steyaert, "A CMOS 18 THz-ohms $240 \mathrm{Mb} / \mathrm{s}$ Transimpedance Amplifier and $155 \mathrm{Mb} / \mathrm{s}$ LED-Driver for Low Cost Optical Fiber Links," IEEE J. Solid-State Cir., Vol. 29, No. 12, pp. 1552-1559, Dec. 1994.

[3] R. Coppoolse, J. Verbeke, P. Lambrecht, J. Codenie, and J. Vandewege, "Comparison of a Bipolar and a CMOS Front End in Broadband Optical Transimpedance Amplifiers," Proc. 38th Midwest Symp. on Cir. and Sys., Brazil, pp. 1026-1029, Aug. 1996.

[4] S.B. Alexander, Optical Communication Receiver Design, SPIE Press, Bellingham, Wash., 1997.

[5] B. Nauta, "A CMOS Transconductance-C Filter Technique for Very High Frequencies," IEEE J. Solid-State Cir, Vol. 27, No. 2, pp. 142-153, Feb. 1992.

[6] M.B. Ritter, F. Gfeller, W. Hirt, D. Rogers, and S. Gowda, "Circuit and System Challenges in IR Wireless Communication," IEEE Int'l Solid-State Cir. Conf., pp. 398-399, San Francisco, CA, Feb. 1996.

[7] R.G. Swartz, Y. Ota, M.J. Tarsia, and V.D. Archer, "A Burst Mode, Packet Receiver with Precision Reset and Automatic Dark Level Compensation for Optical Bus Communications," Symp. on VLSI Technology, Kyoto, Japan, pp. 67-68, May 1993.

[8] A. Tanabe et al., "A Single Chip 2.4 Gb/s CMOS Optical Receiver IC with Low Substrate Crosstalk Preamplifier," IEEE Int'l Solid-State Cir. Conf., pp. 304-305, San Francisco, CA, Feb. 1998.

[9] A.J.C. Moreira, R.T. Valadas, A.M. de Oliveira Duarte, "Characterization and Modelling of Artificial Light Interference in Optical Wireless Communication Systems," IEEE 6th Intl. Symp. on Personal Indoor Mobile Radio Communications, Toronto, Canada, vol.1, pp. 326-331, Sept. 1995. 